\title{
Remote Sensing Satellite Image Database System Allowing Image Portion Retrievals Utilizing Principal Component Which Consists Spectral and Spatial Features Extracted from Imagery Data
}

\author{
Kohei Arai ${ }^{1}$ \\ Graduate School of Science and Engineering \\ Saga University \\ Saga City, Japan
}

\begin{abstract}
Remote Sensing satellite image database which allows image portion retrievals utilizing principal component which consists of spectral and spatial features extracted from the imagery data is proposed. Through the experiments with actual remote sensing satellite images, it is found that the proposed image retrieval does work so well.
\end{abstract}

Keywords_Image retrieval; Spectral and spatial features; $e$

\section{INTRODUCTION}

In accordance with expansion in the multimedia technologies and the Internet, CBIR has been an active research topic since the first 1990's. The concept of content based retrieval (CBR) in image start from the first 1980s and Serious applications started in the first 1990s [1]. Retrieval from databases with a large number of images has attracted considerable attention from the computer vision and pattern recognition society.

Brahmi et al. mentioned the two drawbacks in the keyword annotation image retrieval. First, images are not always annotated and the manual annotation expensive also time consuming. Second, human annotation is not objective the same image may be annotated differently by different observers [2]. Unlike the traditional approach that using the keyword annotation as a method to search images, CBIR system performs retrieval based on the similarity feature vector of color, texture, shape and other image content. Comparing to the traditional systems, the CBIR systems perform retrieval more objectiveness [3].

Global features related to color or texture are commonly used to describe the image content in image retrieval. The problem using global features is this method cannot capture all parts of the image having different characteristics [4]. In order to capture specific parts of the image the local feature is used. The proposed method uses 2D Discrete wavelet transform with Haar base function, combined the two high sub-band frequency to make significant points and edge, choose any part of image by threshold the high coefficient value.
Image feature based image portion retrieval method is proposed in this paper. Principal component which consists of spectral and spatial features extracted from the image are used as features.

The following section describes the proposed image database system followed by some experiments. Then conclusion is described together with some discussions.

\section{Proposed Satellite Image Database System}

\section{A. Image Database}

Database system has to have the following three functions,

1) Image coding and archiving with attributes information

2) Database operations including image retrievals and updating database

(3)Database management including maintenance

Attributes can be used for meta search while meta search allows image retrievals. The meta database is information of image information. Therefore, it is useful for keyword search. For image search, image coding is important. If the image coding is performed perfectly, then image retrievals can be done easily. On the other hands, there is image portion retrievals which allows image portion search in the image in the image database. In the image portion retrievals, some measures for representation of image features are required. One of the simple features is similarity between the template image in the image database and the current query image portions. Other than these, principal component is used as feature. The extracted spectral and spatial features can be projected in principal component space as a vector. The vector can be interpreted as a feature of the image portion in concern and can be used as measure of the similarity between template and the query image portion.

\section{B. Image Retrieval}

Two image retrieval methods are supported by the proposed image retrieval system. One is keyword based search utilizing the following keywords, 


\section{1) Image file name \\ 2) Satellite name \\ 3) Sensor name \\ 4) Band number \\ 5) Area name \\ 6) Observation date \\ 7) Observation time \\ 8) Area feature \\ 9) Cloud content}

Another image retrieval method is image feature based method. There are utilizing features for image retrievals.

\section{Image Feature}

Average, variance, skewness, kurtosis, energy, entropy, the number of edges, minimum/maximum pixel values, contrast, digital count at most frequent pixel values are selected as features. After calculation of features, principal component analysis is applied to the features. The first three principal components are extracted then the mapped features are divided into groups a shown in Figure 1.

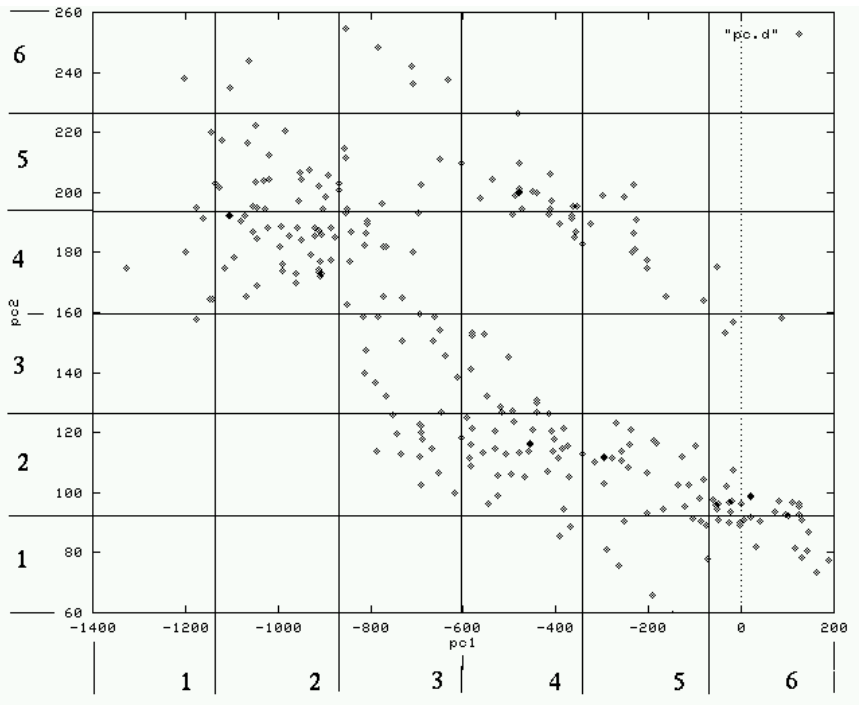

Fig. 1. The first three principal components are extracted then the mapped features are divided into groups

Thus the images in the image database are projected in the principal component space. Then the group number is used for one of attribute information.

\section{Principal Component Analysis}

Let $x_{i}, i=1,2, \ldots, p$ be features extracted from the image assuming correlation among the features. Then new variable, $\mathrm{z}$ is defined as follows,

$$
z=\sum_{i=1}^{p} a_{i} x_{i}
$$

Where $a_{i}$ is determined as maximizing variance of $z$ under the condition of $1=\sum_{i=1}^{p} a_{i}^{2}$. Such this $z$ is called as the first principal component. Also such these coefficients $a_{i}$ are called as the first principal component vector. Rewriting the first principal component as $z_{1}$, and the first principal component vector $a_{l}$, then the arbitrary order, $j$ of principal component $z_{j}$ and principal component vector $a_{j}$ can be written as follows,

$$
\begin{aligned}
& a_{j} a_{j}^{t}=1 \\
& \sigma_{i}^{2} \geq \sigma_{j}^{2}, i \geq j \\
& \rho_{i j}=0, i \neq j
\end{aligned}
$$

\section{E. The Number of Edges}

The following Sobel operator derived edges are extracted from the images for one of the features for image retrievals.

$$
\begin{aligned}
& f_{x}:\left(\begin{array}{ccc}
-1 & 0 & 1 \\
-2 & 0 & 2 \\
-1 & 0 & 1
\end{array}\right) \quad f_{y}:\left(\begin{array}{ccc}
-1 & -2 & -1 \\
0 & 0 & 0 \\
1 & 2 & 1
\end{array}\right) \\
& f_{x}(x, y)= f(i+1, j+1)+2 f(i+1, j) \\
&+f(i+1, j-1)-f(i-1, j+1) \\
&-2 f(i-1, j)-f(i-1, j-1) \\
& f_{y}(x, y)= f(i-1, j-1)+2 f(i, j-1) \\
&+f(i+1, j-1)-f(i-1, j+1) \\
&-2 f(i, j+1)-f(i+1, j+1)
\end{aligned}
$$

Where $f_{x}, f_{v}$ denotes pixel values of edge enhanced images in the horizontal and vertical directions. With appropriated threshold, the horizontal and vertical edges are extracted from the enhanced images. Figure 2 shows one of examples of edge detected images.

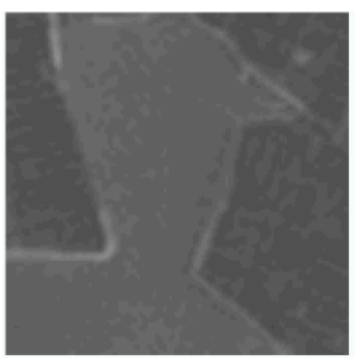

(a)Original image

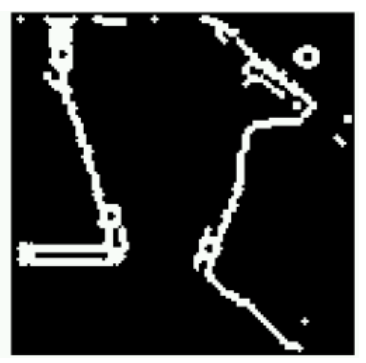

(b)Edge detected image
Fig. 2. Edge detections

\section{F. Texture Features}

The following texture features, mean $(\mu)$ and variance $\left(\sigma^{2}\right)$, skewness (SKW), kurtosis (KRT), energy (EGY), and entropy (ENT) are selected for representation of image features for search. 


$$
\begin{aligned}
\mu & =\sum_{i=o}^{n-1} i P(i) \\
\sigma^{2} & =\sum_{i=0}^{n-1}(i-\mu)^{2} P(i) \\
S K W & =\frac{\sum_{i=o}^{n-1}(i-\mu)^{3} P(i)}{\sigma^{3}} \\
K R T & =\frac{\sum_{i=o}^{n-1}(i-\mu)^{4} P(i)}{\sigma^{4}} \\
E G Y & =\sum_{i=o}^{n-1} P^{2}(i) \\
E P Y & =-\sum_{i=o}^{n-1} P(i) \log P(i)
\end{aligned}
$$

Other than these, the following contrast is also calculated for image retrievals.

$$
C=\frac{I_{\max }-I_{\min }}{I_{\max }+I_{\min }}
$$

\section{G. Principal Component for Image Retrievals}

PCA component derived from satellite imagery data has different variance. In order to normalize the variance, the following equation is used,

$$
x_{i k^{\prime}}=\frac{x_{i k}-\bar{x}_{i}}{\sqrt{s_{i}}} \quad(i=1, \cdots, p),(k=1, \cdots, n)
$$

Where $x_{i i}$ denotes two dimensional imagery of pixel data while $s_{i}$ denotes variance of the pixel data of band $i$. Correlation coefficient between band $\mathrm{i}$ and $\mathrm{j}$ is expressed as follows,

$$
\begin{gathered}
R_{i j}=\frac{s_{i j}}{\sqrt{s_{i i} s_{j j}}} \\
s_{i j}=\frac{1}{n-1} \sum_{k=1}^{n}\left(x_{i k}-\bar{x}_{i}\right)\left(x_{j k}-\bar{x}_{j}\right)
\end{gathered}
$$

Using the correlation coefficients, eigen value and eigen vector of correlation coefficient is estimated utilizing Jacob method. Let $X, \Lambda$ be eigen vector and eigen value of $A$. Non singular matrix $\mathrm{M}$ is utilized, then,

$$
A X=\Lambda X
$$

$$
M A X=\Lambda M X
$$

$y=M X$

$$
M A M^{-1} y=\Lambda y
$$

Thus it is found that eigen value and eigen vector is not going to be changed with the aforementioned operation with the non singular matrix, $M$. The followings are candidates of $M$,

$$
m_{i j}=\left\{\begin{array}{cc}
\cos \theta & (i=j=p, i=j=q) \\
\sin \theta & (i=p, j=q) \\
-\sin \theta & (i=q, j=p) \\
0 & (i \neq j) \\
1 & (i=j)
\end{array}\right.
$$

These are called as basic orthogonal matrices. Using the basic orthogonal matrix, power of A can be written as follows,

$$
A^{(s+1)}=M^{\prime} A^{(s)} M \quad(s=1,2,3, \cdots)
$$

Namely,

$$
\begin{aligned}
& a_{i j}^{(s+1)}=a_{i j}^{(s)} \cos \theta+a_{i k}^{(s)} \sin \theta \\
& a_{i k}^{(s+1)}=-a_{i j}^{(s)} \sin \theta+a_{i k}^{(s)} \cos \theta \\
& a_{j j}^{(s+1)}=a_{j j}^{(s)} \cos ^{2} \theta+2 a_{j k}^{(s)} \cos \theta \sin \theta+a_{k k}^{(s)} \sin ^{2} \\
& a_{k k}^{(s+1)}=a_{j j}^{(s)} \sin ^{2} \theta-2 a_{i k}^{(s)} \cos \theta \sin \theta+a_{k k}^{(s)} \cos ^{2} \\
& a_{j k}^{(s+1)}=\left(a_{k k}^{(s)}-a_{j j}^{(s)}\right) \cos \theta \sin \theta+a_{j k}^{(s)}\left(\cos ^{2} \theta-\sin ^{2} \theta\right)
\end{aligned}
$$

If the maximum absolute value of the non diagonal elements, $a_{i j}^{(s)}$ is found, then

$$
\begin{aligned}
& \left(a_{k k}^{(s)}-a_{j j}^{(s)}\right) \cos \theta \sin \theta+a_{j k}^{(s)}\left(\cos ^{2} \theta-\sin ^{2} \theta\right)=0 \\
& \left(a_{j j}^{(s)}-a_{k k}^{(s)}\right) \sin 2 \theta=2 a_{j k}^{(s)} \cos 2 \theta \\
& \tan 2 \theta=\frac{2 a_{j k}^{(s)}}{\left(a_{j j}^{(s)}-a_{k k}^{(s)}\right)} \quad\left(-\frac{\pi}{4} \leq \theta \leq \frac{\pi}{4}\right)
\end{aligned}
$$

It is possible to find the $\theta$ as to equation (12) is satisfied. Then,

$$
A^{(s)}=M_{s}^{\prime} \cdots M_{1}^{\prime} A M_{1} \cdots M_{s}
$$

Thus the eigen values are obtained,

$$
A^{(s)} \rightarrow \Lambda
$$

If we choose Mi as follows,

$M=M_{1} \cdots M_{s}$

Then,

$$
M^{\prime} A M=\Lambda
$$

$A M=M \Lambda$

Thus eigen values are determined followed by the corresponding eigen vector.

\section{H. Implementation of Image Retrievals}

Web design for image retrievals is conducted. Figure 3 shows the registration form. The aforementioned key words for key word image retrievals can be input to the database. 


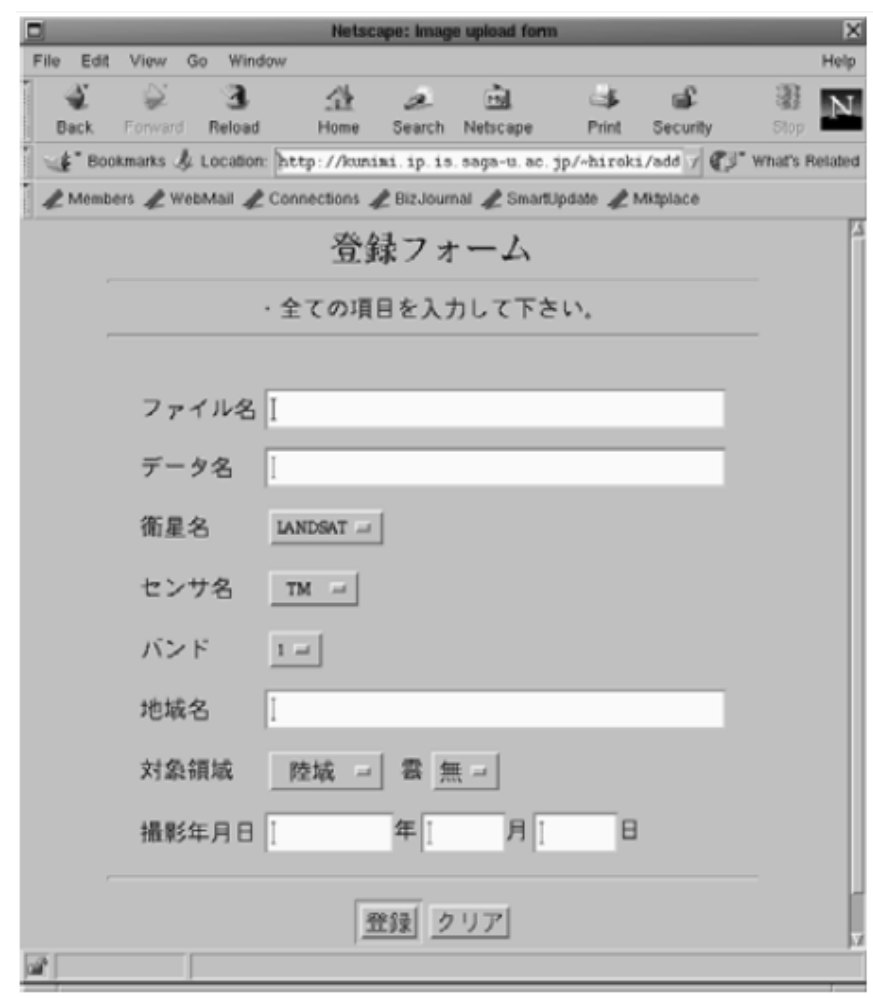

Fig. 3. Registration for which allows input keywords through dialog boxes

The registered image with attribution data can be confirmed as shown in Figure 4. Figure 5 shows top page of the image retrieval system. If user input query information through the web page which is shown in Figure 6, then search results can be obtained from the image retrieval system as shown in Figure 7. Keyword search can be done through the web page of Figure 6.

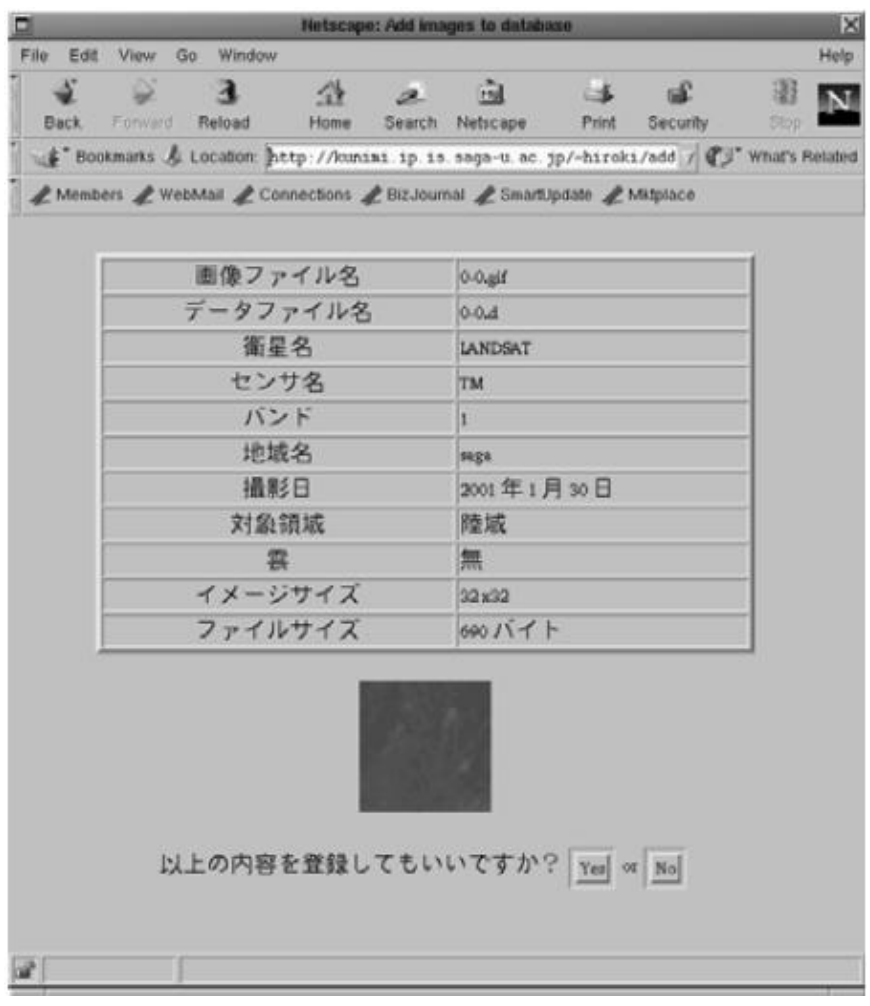

Fig. 4. Confirmation of the registered image and attribution data

Image feature based search is also done through the web page of Figure 8 . Then the search results can be obtained as shown in Figure 9. Also the attribution information is obtained as shown in Figure 10. 


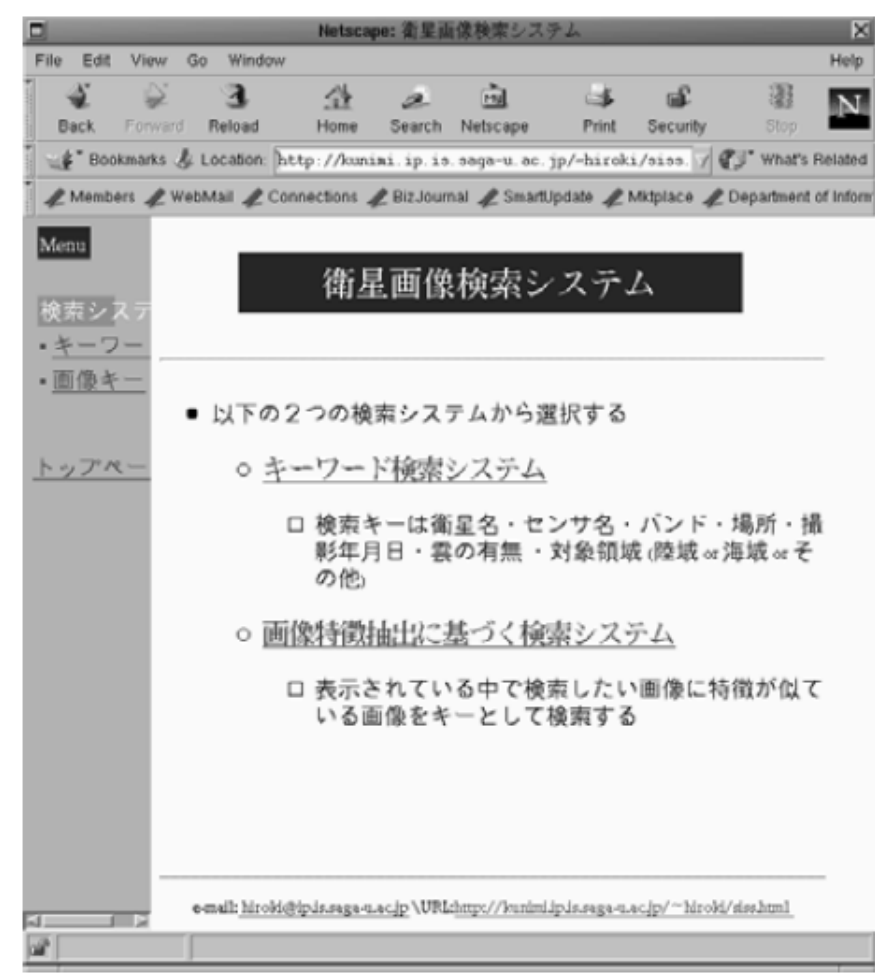

Fig. 5. page of the image retrieval system

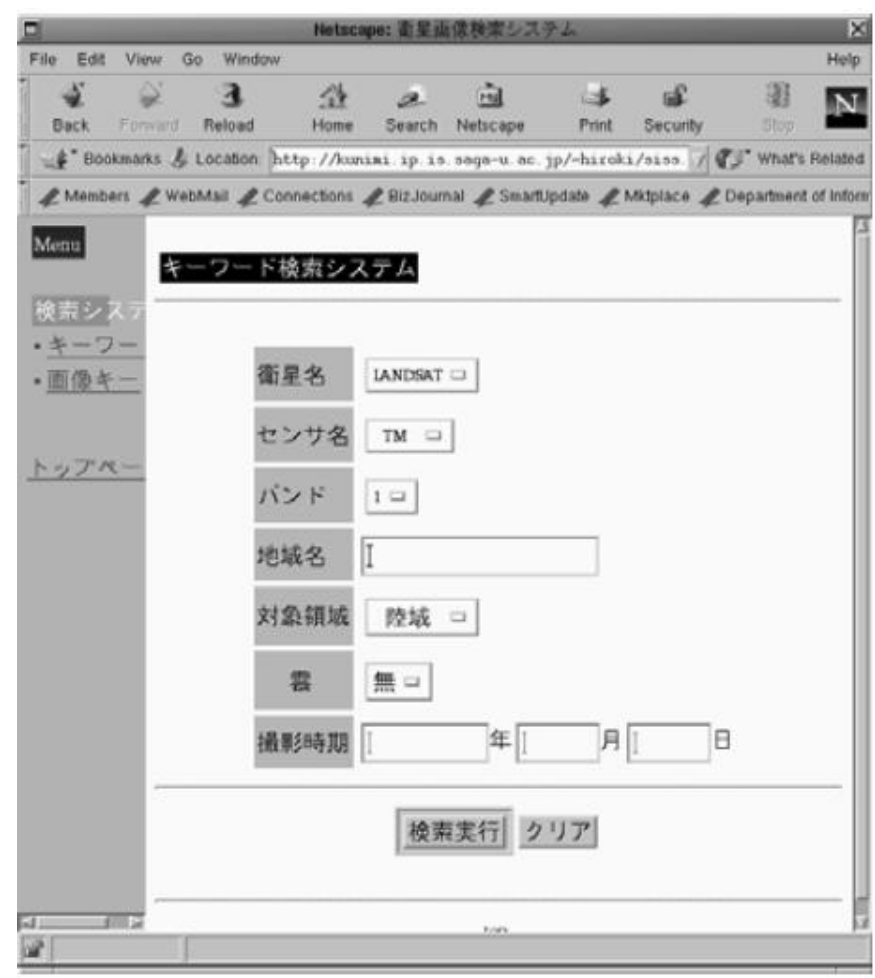

Fig. 6. Query information can be input through the web page

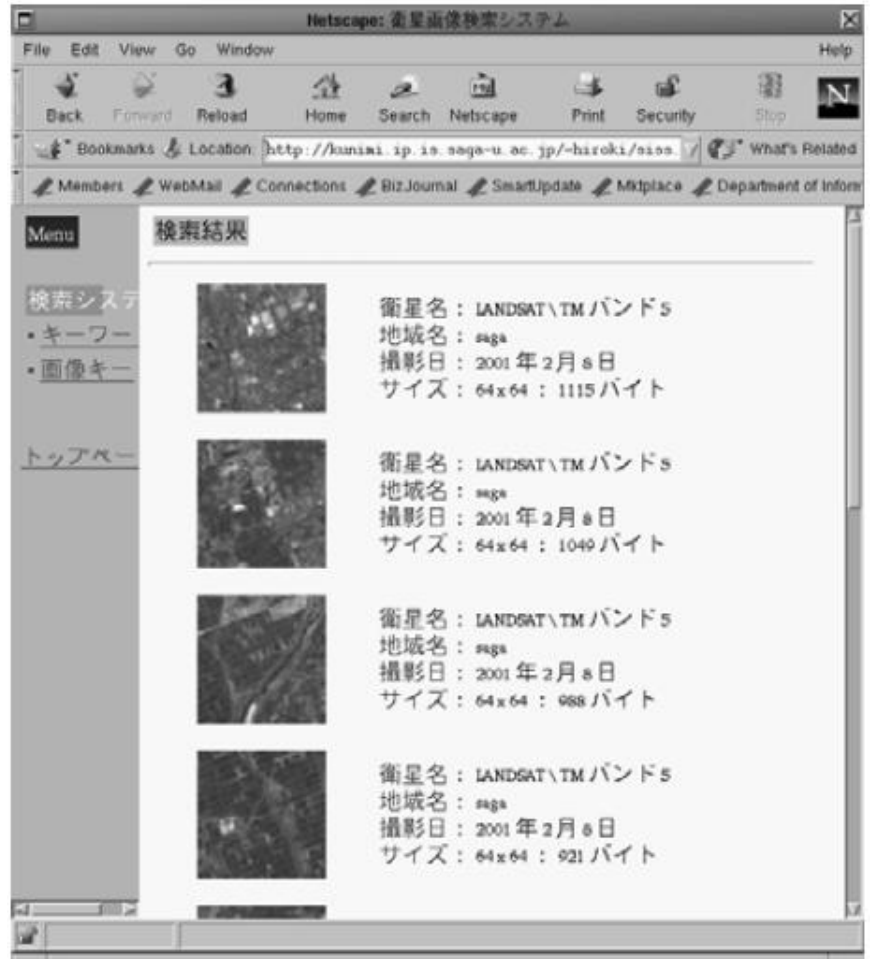

Fig. 7. Retrieved image are obtained as candidates

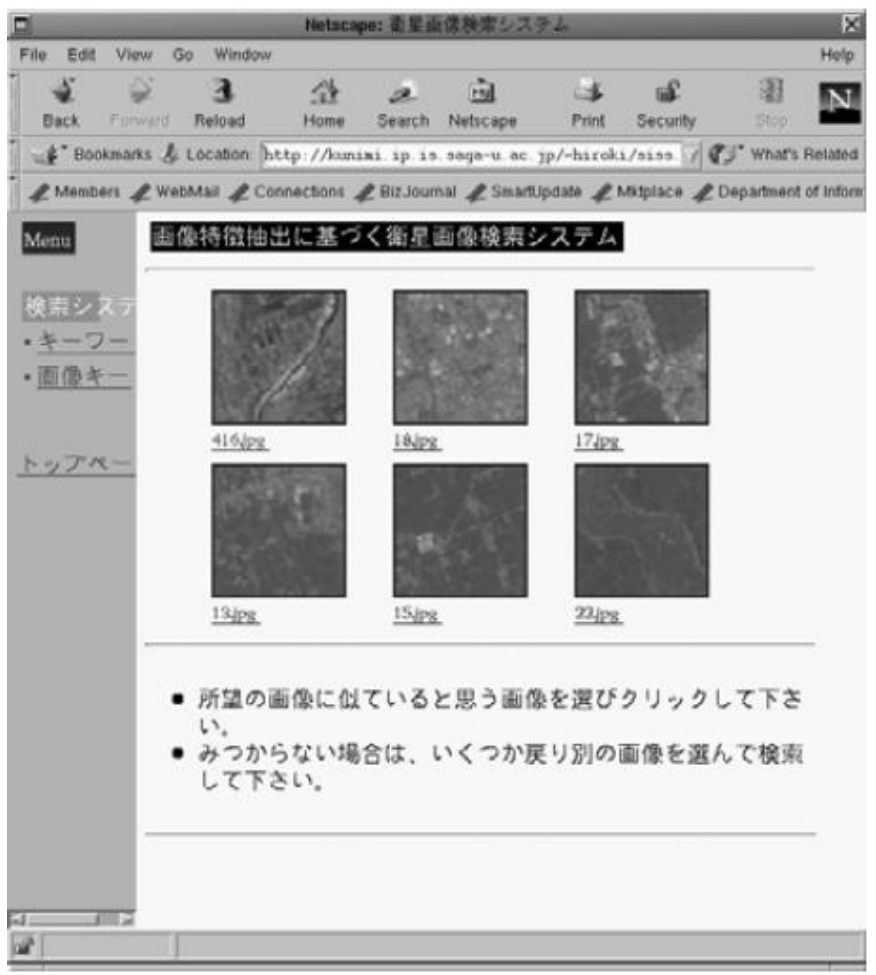

Fig. 8. Image feature based search is also done through the web page. 


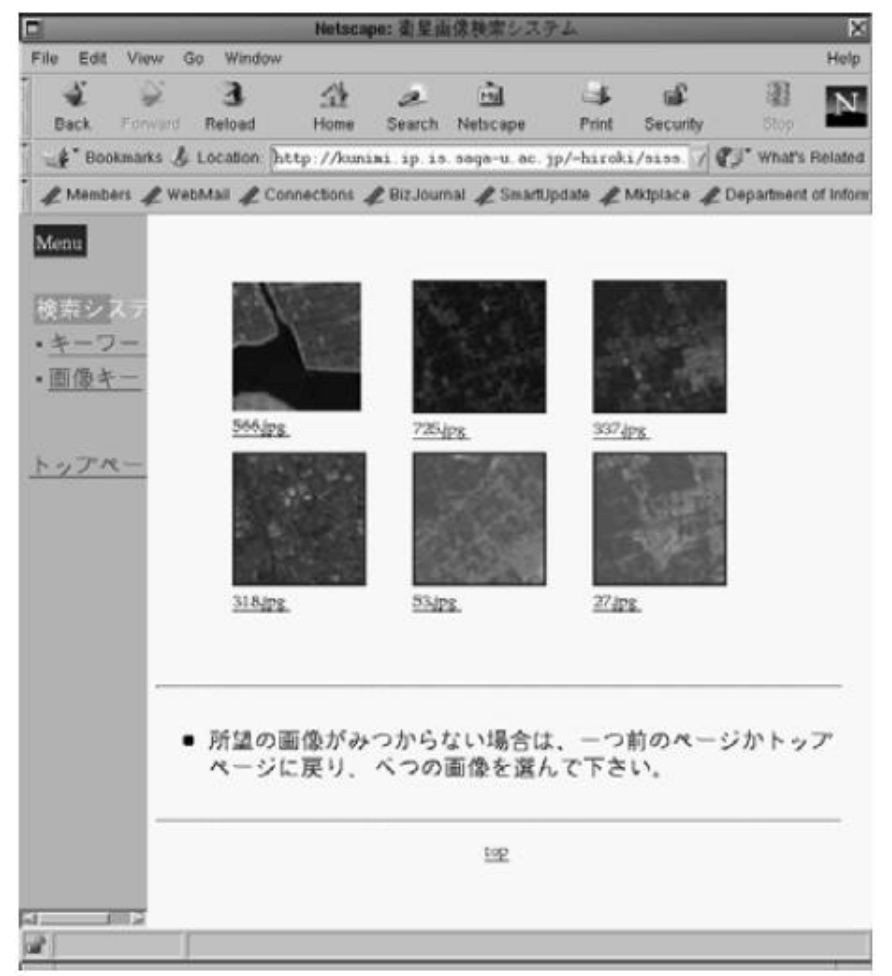

Fig. 9. Search results can be obtained

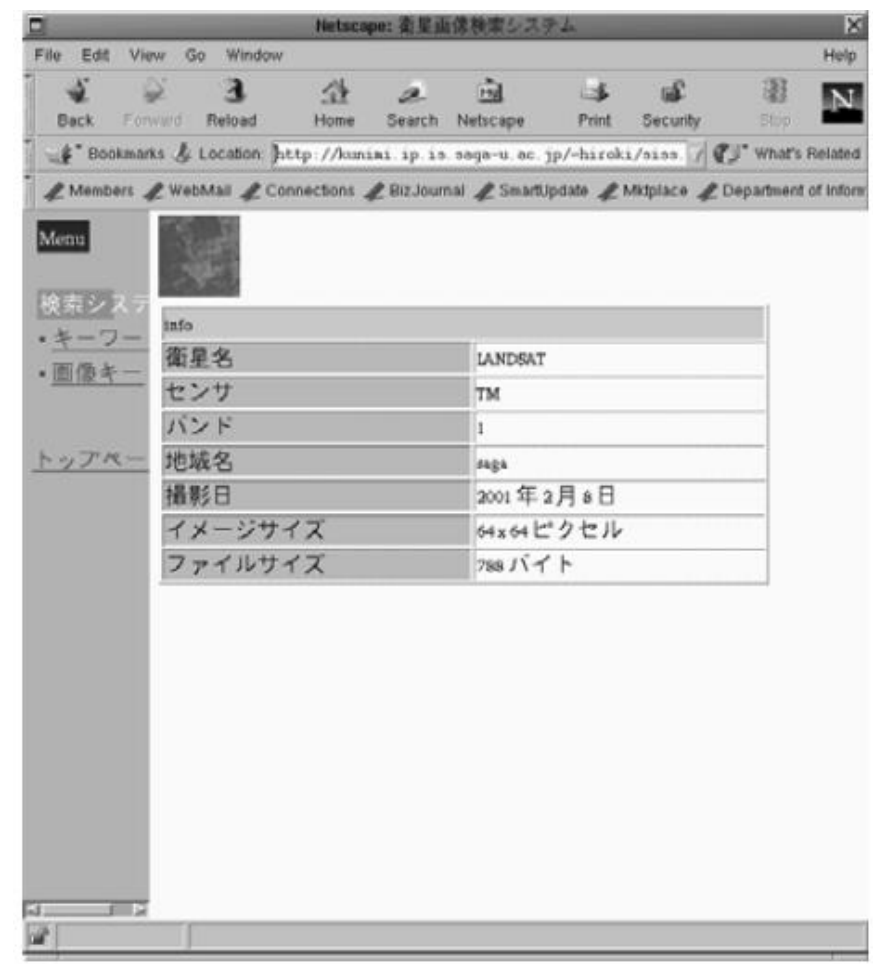

Fig. 10. Attribution information is obtained

\section{EXPERIMENTS}

\section{A. A. Data Used and Evaluation of Hit Ratio and the Number of Steps for Search}

Landsat Thematic Mapper: TM data are used for experiments. From the TM data, 288 of 64 by 64 pixels of sample images with the different types of image features are selected.

12 students are nominated for performance tests of hit ratio and the required number of steps for image retrievals.

\section{B. B. Image Retrieved Results}

The examples of the first principal component images (the largest eigen vector which corresponds to the largest eigen value) are shown in Figure 11.
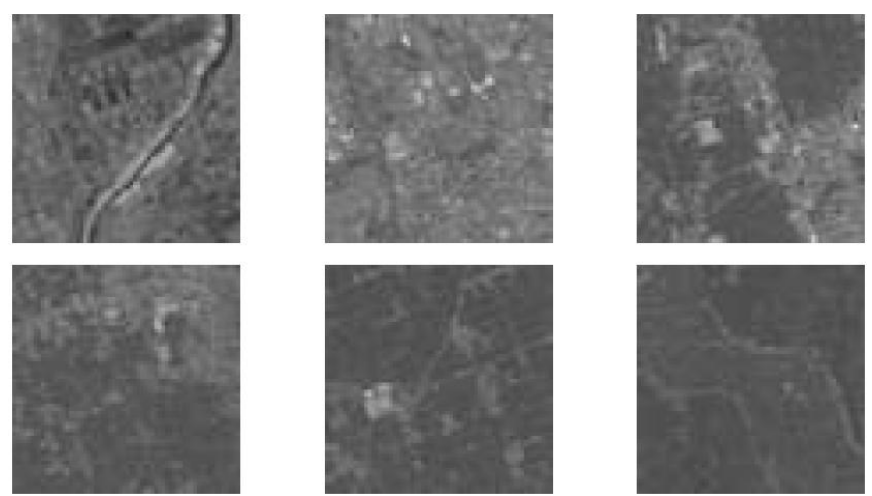

Fig. 11. Examples of the first principal component images (the largest eigen vector which corresponds to the largest eigen value)

Image feature based image retrieval is conducted. If user clicks one of the displayed image of Figure 11, then the retrieved image candidates are displayed in accordance with the distance between the query image and the candidate images as shown in Figure 12. Figure 12 shows the example images extracted from the first group of the first principal component images. It is found that image complexity can be expressed with the first principal component images.
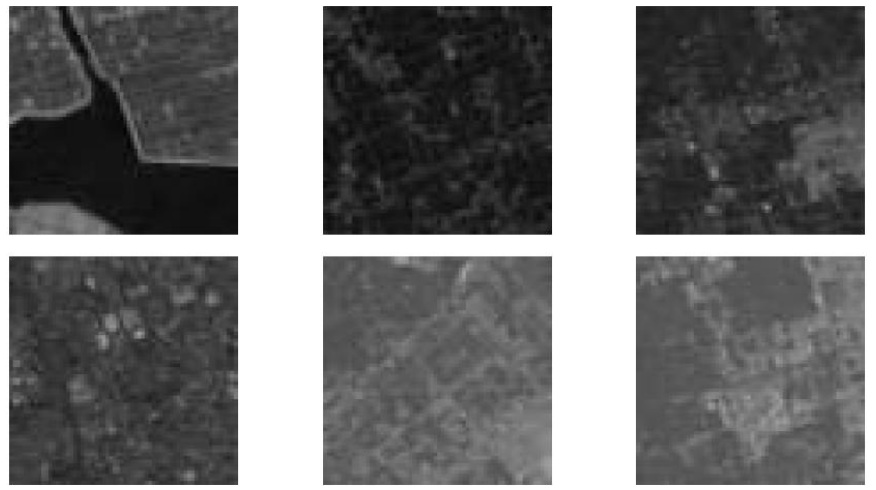

Fig. 12. Example images extracted from the first group of the first principal component images. 


\section{Evaluation of Hit Ratio and the Required Number of Steps for Search}

By using the query images of Figure 13, image feature based image retrievals are conducted by 12 students who do not have any experience and knowledge about satellite imagery data at all. The evaluation results are shown in Table 1 .

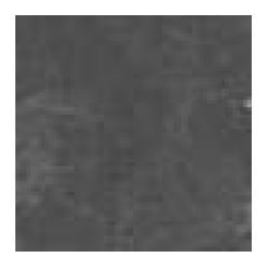

A

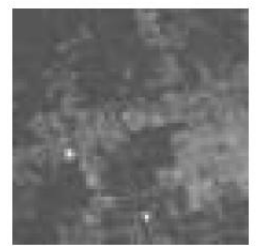

D

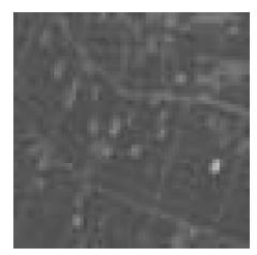

B

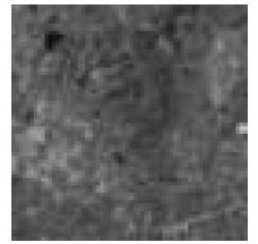

$\mathrm{E}$

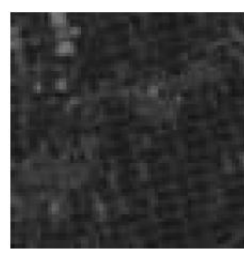

C

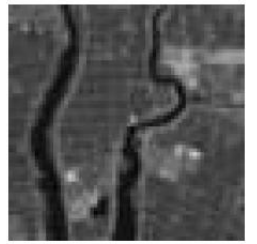

F
Fig. 13. Query images which are selected from the 288 of sample Landsat TM images in the image database.

TABLE I. HIT RATIO AND THE REQUIRED NUMBER OF STEPS FOR SEARCH WHICH ARE EVALUATED WITH LAND SAT SATELLITE TM IMAGERY DATA BY 12 STUDENTS.

\begin{tabular}{|c|r|r|r|r|r|c|}
\hline & \multicolumn{1}{|c|}{ A } & B & \multicolumn{1}{c|}{ C } & D & E & F \\
\hline Hit Ratio (\%) & 54.5 & 36.4 & 18.2 & 72.7 & 9.1 & 100 \\
\hline No.of Steps & 5.7 & 12 & 21 & 6.4 & 3 & 4.1 \\
\hline
\end{tabular}

Hit ratio and the required number of steps for search are totally depending on the complexity of the query image. The highest hit ratio is shown for the query image " $\mathrm{F}$ " while the lowest hit ratio is shown for the query image of "E". Therefore it is said that image feature rich image of " $F$ " is easy to retrieve while image feature poor image of " $\mathrm{E}$ " is difficult for search. On the other hands, the number of steps required for image retrievals for the query image " $\mathrm{C}$ " is greatest and that for the query image " $\mathrm{E}$ " is smallest. This implies that it takes long time to search the query image "C", at the same time, not so large hit ratio cannot be achieved for such query image. Meanwhile, user tried to search images for the query image "E", and then user quit to search because it is difficult to search. Therefore, not only hit ratio but also the number of steps required for search is poor.

\section{CONCLUSION}

Remote Sensing satellite image database which allows image portion retrievals utilizing principal component which consists of spectral and spatial features extracted from the imagery data is proposed. Through the experiments with actual remote sensing satellite images, it is found that the proposed image retrieval does work so well.

Experimental results show that hit ratio depends on the image features which are containing in the satellite image in concern. The required number of steps does not depend on the image features which are contained in the satellite image in concern. Because user forgets about the image retrievals when they feel that it is difficult to search due to the fact that satellite image in concern does not have any specific features.

\section{ACKNOWLEDGMENT}

The author would like to thank Mr.Hiroki Fujikawa for his effort to conduct experiments.

\section{REFERENCES}

[1] Henning M"uller, Antoine Geissbuhler, et al. "Benchmarking Image Retrieval Applications". San Francisco, CA: In The Seventh International Conference, September 2004. pp. 334-337.

[2] Kherfi, M., Brahmi, D. and Ziou D. "Combining Visual Features with Semantics for a More Effective Image Retrieval". : in ICPR '04, vol. 2, 2004. pp. 961-964.

[3] H. Yu, M. Li, H.-J. Zhang and Feng, J. "Color texture moments for content-based image retrieval". : In International Conference on Image Processing, 2002. pp. 24-28.

[4] N. Sebe, Q. Tian, E. Loupias, M. Lew and T. Huang. "Evaluation of salient point techniques". s.1. : In International Conference, 2004.

\section{AUTHORS PROFILE}

Kohei Aarai He received BS, MS and PhD degrees in 1972, 1974 and 1982, respectively. He was with The Institute for Industrial Science and Technology of the University of Tokyo from April 1974 to December 1978 and also was with National Space Development Agency of Japan from January, 1979 to March, 1990. During from 1985 to 1987, he was with Canada Centre for Remote Sensing as a Post Doctoral Fellow of National Science and Engineering Research Council of Canada. He moved to Saga University as a Professor in Department of Information Science on April 1990. He was a councilor for the Aeronautics and Space related to the Technology Committee of the Ministry of Science and Technology during from 1998 to 2000. He was a councilor of Saga University for 2002 and 2003. He also was an executive councilor for the Remote Sensing Society of Japan for 2003 to 2005. He is an Adjunct Professor of University of Arizona, USA since 1998. He also is Vice Chairman of the Commission-A of ICSU/COSPAR since 2008. He wrote 30 books and published 332 journal papers. 